\title{
Models for photoabsorption cross section estimates
}

\author{
V. Plujko ${ }^{1, a}$, I. Kadenko ${ }^{1}$, S. Goriely ${ }^{2}$, E. Kulich ${ }^{1}$, O. Davidovskaya ${ }^{3}$, and O. Gorbachenko ${ }^{1}$ \\ 1 Nuclear Physics Department, Taras Shevchenko National University, 03022 Kyiv, Ukraine \\ 2 Institut d'Astrophysique Université Libre de Bruxelles, Brussels, Belgium \\ 3 Nuclear Structure Department, Institute for Nuclear Research, NAS of Ukraine, Kyiv, Ukraine
}

\begin{abstract}
Extensive comparisons of dipole radiative strength calculations within the framework of different methods are performed in middle and heavy atomic nuclei both near and out of the beta-stability line. Photoabsorption cross sections are calculated and compared with experimental data to test radiative strength function (RSF) models. It was found that phenomenological closed-form models of modified Lorentzian can be recommended for overall estimates of the dipole RSF in the energy region up to about $20 \mathrm{MeV}$, at least for nuclei for which experimental giant dipole resonance (GDR) parameters exist or the corresponding systematics can be adopted. Otherwise, the HFB-QRPA microscopic method and semi-classical approach with moving surface appear to be more adequate for the determination of the dipole RSF.
\end{abstract}

\section{Introduction}

The radiative strength function (RSF) is an important ingredient in the statistical theory of nuclear reactions [1-3]. In particular, the photoabsorption cross section of dipole gamma-rays with energy $E_{\gamma}$ by a cold spherical nucleus is proportional to the RSF of the photoabsorption process $\left(\vec{f}\left(E_{\gamma}\right)\right)$ and given by the relation:

$$
\sigma\left(E_{\gamma}\right)=3(\pi \hbar c)^{2} \cdot E_{\gamma} \cdot \vec{f}\left(E_{\gamma}\right)
$$

The calculation of nuclear reaction ingredients is, as a rule, a rather time consuming procedure and simple closed-form expressions are often used to estimate input parameters. The electric dipole (E1) gamma-transitions tend to dominate in nuclear processes. Different analytical expressions were recently proposed to describe the E1 RSF in middle and heavy atomic nuclei (see refs. [2,3]).

In this contribution, different models of the photoabsorption cross sections are tested. More specifically, experimental photoabsorption data are compared with theoretical calculations performed within the framework of the microscopic Hartree-Fock-Bogoliubov plus quasi-particle random phase approximation model (HFB-QRPA) [4,5], the semi-classical approach with moving surface (MSA) [6] and the more traditional Lorentzian-type models using closed-form expressions for dipole RSF [2,3]. Here we also consider the description of the dipole photo-excitation RSF within two simplified versions of the Lorentzian model with energy dependent widths in the form of a power series in $E_{\gamma}$.

\section{Main features of the tested RSF models}

Different models have been developed to describe the dipole RSF microscopically. We consider here the expressions for the linear response function of an atomic nucleus to

\footnotetext{
${ }^{a}$ Presenting author, e-mail: plujko@univ.kiev.ua
}

an electric dipole field within the framework of the HFBQRPA approach with Skyrme forces and an additional folding procedure introduced to take collisional damping into account $[4,5]$.

Tested semi-classical MSA method [6] is based on solving the kinetic Landau-Vlasov equation for finite system with a moving surface [7]. In this case, the nuclear response function (and the RSF) consists of two terms, namely a volume component that is related to the shift of proton and neutron fluids in the nuclear interior and a surface component due to vibrations of mutually non-penetrating neutron and proton spheres.

The absorption of dipole gamma-rays in the $E_{\gamma} \leq 20 \mathrm{MeV}$ energy region is mainly governed by excitation of the isovector giant dipole resonance (GDR). Therefore the phenomenological expressions of the RSF for spherical nuclei in this energy range (see $[2,3]$ and reference therein) have a Lorentzian shape (in units of $\mathrm{MeV}^{-3}$ ):

$$
\vec{f}\left(E_{\gamma}\right)=8.674 \cdot 10^{-8} \sigma_{r} \Gamma_{r} \frac{E_{\gamma} \Gamma\left(E_{\gamma}\right)}{\left(E_{\gamma}^{2}-E_{r}^{2}\right)^{2}+\left(E_{\gamma} \Gamma\left(E_{\gamma}\right)\right)^{2}} .
$$

Here, $\Gamma_{r}$ and $E_{r}$ are width and energy of the GDR (in MeV); $\sigma_{r}$ is the peak photoabsorption cross section (in mb); $\Gamma\left(E_{\gamma}\right)$ is an energy-dependent "width" that is equal to the GDR width at $E_{\gamma}=E_{r}$ :

$$
\Gamma_{r}=\Gamma\left(E_{\gamma}=E_{r}\right) .
$$

Phenomenological models of the RSF differ in the expressions for $\Gamma\left(E_{\gamma}\right)$ which reflects the description of the collective state damping. In the standard Lorentzian model (SLO $[8,9]$ ), the width $\Gamma\left(E_{\gamma}\right)$ is taken as an energy-independent quantity equal to the GDR width $\Gamma_{r}$. The width shape is similar to the fragmentation component of the collective excitation width (one-body dissipation) and corresponds to the nucleon collisions with a moving surface of the self-consistent meanfield. In this case, the contribution of nucleon collisions in the nuclear interior is not included [10]. 


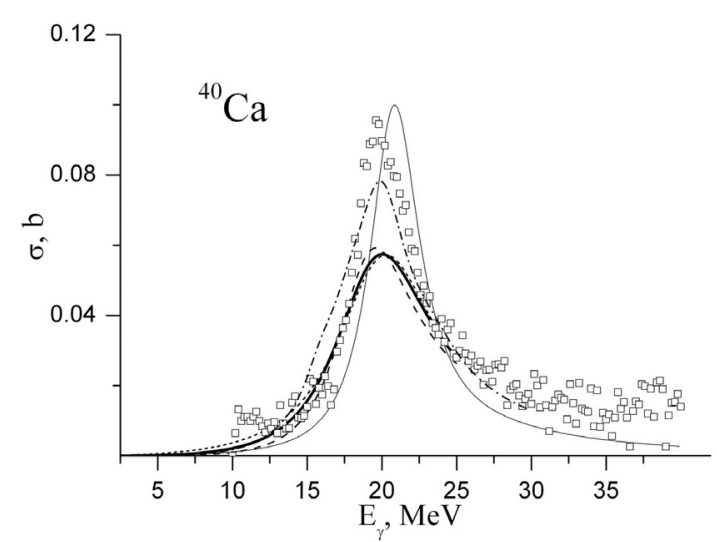

Fig. 1. Comparison of calculated photoabsorption cross sections for ${ }^{40} \mathrm{Ca}$. Curves: MLO1 (thick solid line), SLO (dotted line), EGLO (dashed line), HFB-QRPA (dash-dot line), MSA (thin solid line); experimental data are taken from [17].

In the enhanced generalized Lorentzian model (EGLO $[2,3,11,12])$, the width $\Gamma\left(E_{\gamma}\right)$ depends on the gamma-ray energy. The EGLO-width at $E_{\gamma}=E_{r}$ is proportional to the collisional component of the zero sound damping width in the infinite Fermi liquid that corresponds to the two-body (collisional) relaxation only.

The width $\Gamma\left(E_{\gamma}\right)$ also depends on the energy in both the modified Lorentzian models (MLO1, MLO2, MLO3 [3,13, 14]) and the generalized Fermi-liquid model (GFL $[3,15])$. The collisional and fragmentation contributions are included in the width expression. These RSF models are recognized by the width expressions due to slightly different assumptions on the damping mechanism of collective states.

We also consider the description of the dipole photoexcitation RSF within two simplified versions of the modified Lorentzian model. Namely, the widths $\Gamma\left(E_{\gamma}\right)$ in these approaches are taken as a power of series in $E_{\gamma}$ of the first (SMLO1) and second (SMLO2) degree:

$$
\begin{gathered}
\Gamma\left(E_{\gamma}\right)=a \cdot E_{\gamma}, \\
\Gamma\left(E_{\gamma}\right)=b_{0}+b_{1} \cdot E_{\gamma}+b_{2} \cdot E_{\gamma}^{2} .
\end{gathered}
$$

For $a=\Gamma_{r} / E_{r}, b_{0}+b_{1} \cdot E_{r}+b_{2} \cdot E_{r}^{2}=\Gamma_{r}$ and gamma-ray energies from zero till energies of the GDR, these quantities approximately correspond to the MLO1 and MLO2 widths, respectively. The parameters $a,\left\{b_{j}\right\}$ are obtained by fitting experimental data on photoabsorption cross sections of spherical nuclei (listed in ref. [22]). The ready-to-use tables of the $a$, $\left\{b_{j}\right\}$ values and the related GDR parameters will be published somewhere else. The GDR parameters within SMLO models have nearly the same values as in the RIPL-2 library with difference of no more than $\sim 10 \%$.

\section{Calculation results and discussion}

The E1 photoabsorption cross sections were calculated for spherical even-even nuclei and the results for ${ }^{40} \mathrm{Ca},{ }^{208} \mathrm{~Pb}$ as well as their comparison with experimental data are shown in figures 1-3.

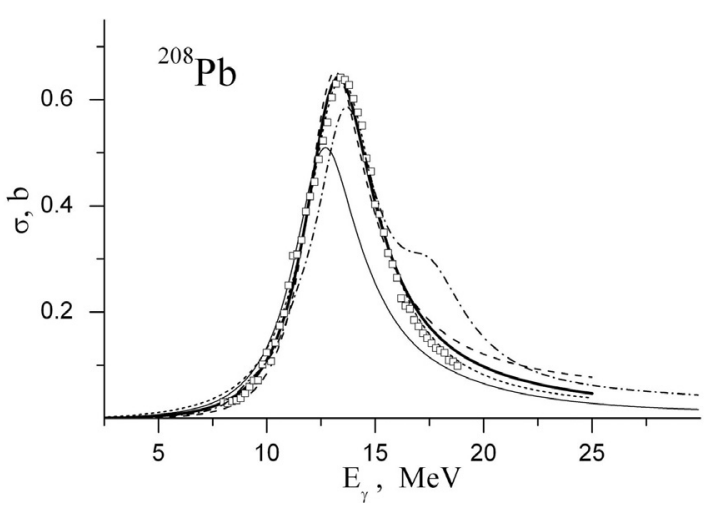

Fig. 2. Comparison of calculated photoabsorption cross sections for ${ }^{208} \mathrm{~Pb}$. Denotations of the curves are the same as in figure 1 ; experimantal data are taken from [18].

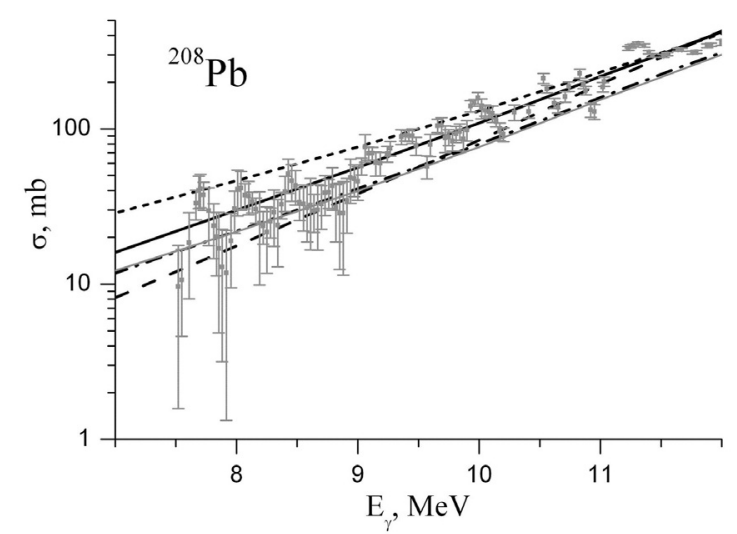

Fig. 3. Low-energy part of photoabsorption cross sections for ${ }^{208} \mathrm{~Pb}$ in low energy region. Curves: MLO1 (solid line), SMLO1 (dasheddotted line), EGLO (dashed line), SLO (dotted line), GFL (thin solid line); experimental data from [18].

The sets of the GDR parameters from RIPL-2 [3] are used in calculations within the MLO, SLO, EGLO models. The following values of the parameters of the MSA model were used: the Fermi energy $36.9 \mathrm{MeV}$; effective nucleon mass $m^{*}=0.9$; the Landau parameters $F_{0}^{\prime}=0.929, F_{0}=-0.227$ for the isovector and isoscalar components of the nucleon interaction in symmetric nuclear matter; the symmetry surface energy is $\mathrm{Q}=70 \mathrm{MeV}$. The relaxation time approximation was used for the collision integral with a constant value of the collective state relaxation time corresponding to the systematics of the GDR widths.

Trends of photoabsorption cross sections with different variants of MLO and SMLO approach (as well as GFL model) are similar. Therefore, only the MLO1 calculations will be shown in figures. The results due to different variants of SMLO are very close to each other but the SMLO2 approach provides slightly better description of the dipole RSF. The values of the least-square deviation between the SMLO calculations and experimental data are nearly twice smaller than for SLO.

In agreement with our previous investigations [3,19,20], the closed-form methods of the RSF calculations describe the photoabsorption cross sections for medium-mass and 

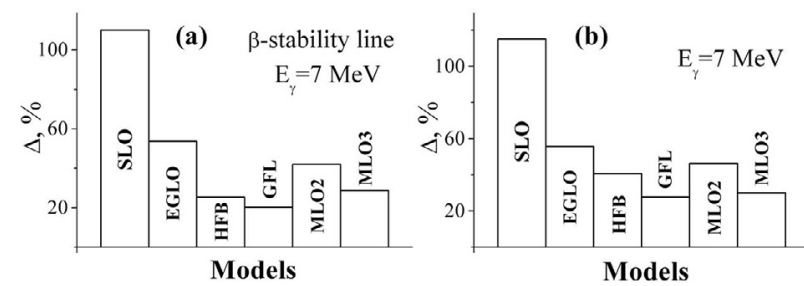

Fig. 4. Relative deviation of RSF calculated within different models with respect to the MLO1 predictions for nuclei on $\beta$-stability line (a) and for isotopes between neutron and proton drip-lines (b) at $E_{\gamma}=7 \mathrm{MeV}$.
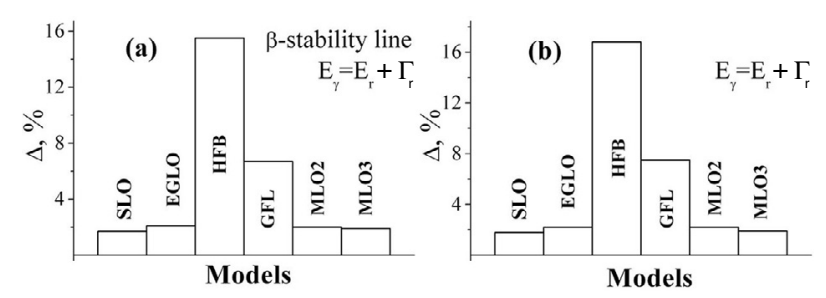

Fig. 5. Same as figure 4, but for the energy $E_{\gamma}=E_{r}$.
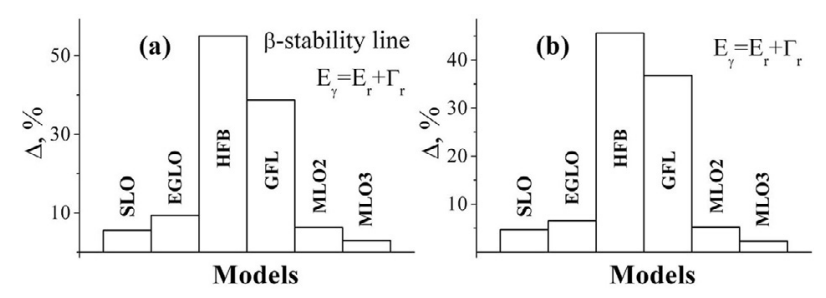

Fig. 6. Same as figure 4, but for the energy $E_{\gamma}=E_{r}+\Gamma_{r}$.

heavy nuclei somewhat better than global semi-microscopic methods at least when experimental values of the GDR parameters or values from reliable systematics are available. The different closed-form expressions for RSF gives rather similar cross sections at gamma-ray energies in the vicinity of the GDR energy, but differ away from the cross section peak. The calculations within the MLO, SMLO and GFL models at gamma-ray energies close to neutron separation energy are in better accordance with experimental data than calculations within SLO and EGLO models. More specifically, the EGLO model somewhat underestimates and SLO model overestimates the experimental data. The calculations within the HFB-QRPA lead in many cases to low photoabsorption cross sections in the GDR energy region $E_{\gamma}=E_{r}$. On average, both the MSA and HFB-QRPA calculations agree with the experimental data and even gives better predictions for light nuclei (like ${ }^{40} \mathrm{Ca}$, see fig. 1) as compared with the the closed-form approaches.

Figures 4-6 present the relative deviations $\Delta$ of the photoabsorption cross section calculated within different methods with respect to the MLO1 prediction:

$$
\Delta=\left[\frac{1}{N_{\max }} \sum_{i=1}^{N_{\max }}\left[\frac{\sigma\left(A_{i}, \text { Model }\right)-\sigma\left(A_{i}, \text { MLO1 }\right)}{\sigma\left(A_{i}, M L O 1\right)}\right]^{2}\right]^{1 / 2},
$$

where $N_{\max }$ is amounts of atomic nuclei with mass-numbers $A_{i}$ involved in the calculations.
The relative deviations are compared at three gamma-ray energies: 1) close to neutron separation energy $\left(E_{\gamma}=7 \mathrm{MeV}\right)$ in figure $4 ; 2)$ at the GDR energy $\left(E_{\gamma}=E_{r}\right)$ in figure 5 and 3$)$ at an energy exceeding the GDR energy, namely $\left(E_{\gamma}=E_{r}+\Gamma_{r}\right)$ in figure 6. The systematics $E_{r}=31.2 / A_{i}^{1 / 3}+$ $20.6 / A_{i}^{1 / 6}, \Gamma_{r}=0.026 E_{r}^{1.9}$ (in MeV) are adopted to estimate $E_{r}$ and $\Gamma_{r}$. The calculations were performed for atomic nuclei both on the beta-stability line (panels (a) in figures 4-6) and with allowance of the isotopes out of this range (panels (b)). In the last case the calculations were performed for the 3317 nuclei with $8 \leq \mathrm{Z} \leq 84$ lying between the proton and neutron drip-lines and listed at the website of ref. [4]. The approximation of axially deformed nuclei was used for the RSF calculations in deformed nuclei [3].

Numerical studies indicate that the calculations of the radiative strength functions within the closed-form models lead to similar results in a range of $\gamma$-ray energies around the GDR peak. However the results within MLO and EGLO models differ from SLO predictions in the low energy region. In particular, at $E_{\gamma}=7 \mathrm{MeV}$ the RSF within the SLO approach is about twice larger with respect to the one obtained within the MLO and EGLO models (excluding the atomic mass range $A \in 150 \div 180$ for EGLO model).

The overall comparison of the calculations within different models and experimental data show that MLO, SMLO and GFL approaches provide the most reliable simple methods to determine the E1 RSF over a relatively wide energy interval ranging from zero to above the GDR peak, at least for nuclei for which experimental GDR parameters exist or the corresponding systematics can be safely applied to.

The MLO, SMLO and GFL calculations are not time consuming and can be recommended for general use; any of them can be used to estimate the photoabsorption cross sections and to extract the GDR parameters from experimental data for medium or heavy nuclei. It should be noted that a collisional component of the GFL damping width can become negative in some deformed nuclei. It was found (see fig. 1) that the HFBQRPA [4,5] and semi-classical MSA [6] seem to be more adequate for the description of the dipole RSF in spherical nuclei of medium mass, especially if reliable values of the giant dipole resonance parameters are not experimentally available.

This work is supported in part by the IAEA(Vienna) under IAEA Research Contract No. 12492.

\section{References}

1. M.A. Lone, in Neutron induced reactions. Proc. 4th International Symposium, Smolenice, Czechoslovakia, 1985, edited by J. Kristiak, E. Betak, D. Reidel. (Dordrecht, Holland, 1986), p. 238 .

2. J. Kopecky, in Handbook for calculations of nuclear reaction data. Reference Input Parameter Library (RIPL). IAEATEDOC-1034. 1998. Ch. 6; http://www-nds.iaea.or.at/ripl/.

3. T. Belgya, O. Bersillon, R. Capote, T. Fukahori, G. Zhigang, S. Goriely, M. Herman, A.V. Ignatyuk, S. Kailas. A. Koning, P. Oblozinsky, V. Plujko, P. Young. IAEA-TECDOC-1506: Handbook for calculations of nuclear reaction data: Reference Input 
Parameter Library-2, IAEA, Vienna, 2005, Ch. 7; http://wwwnds.iaea.org/RIPL-2/.

4. S. Goriely, E. Khan, Nucl. Phys. A 706, 217 (2002); http://wwwastro.ulb.ac.be/Html/gamma.html.

5. S. Goriely, E. Khan, M. Samyn, Nucl. Phys. A 739, 331 (2004).

6. V.I. Abrosimov, O.I. Davidovskaya, Izvestiya RAN. Seriya Fiz. 68, 200 (2004); Ukrainian Phys. Jour. 53, 234 (2006).

7. V.I. Abrosimov, M. Di Toro, V.M. Strutinsky, Nucl. Phys. A 562 , 41 (1993).

8. D.M. Brink, Ph.D. thesis, Oxford University, 1955.

9. P. Axel, Phys. Rev. 126, 671 (1962).

10. V.M. Kolomietz, V.A. Plujko, S. Shlomo, Phys. Rev. C 54, 3014 (1996).

11. J. Kopecky, M. Uhl, Phys. Rev. C 41, 1941 (1990).

12. J. Kopecky, M. Uhl, R.E. Chrien, Phys. Rev. C 47, 312 (1993).

13. V.A. Plujko, Nucl. Phys. A 649, 209 (1999); Acta Phys. Pol. B 31, 435 (2000).
14. V.A. Plujko, S.N. Ezhov, M.O. Kavatsyuk et al., J. Nucl. Sci. Technol. 2, 811 (2002).

15. S.F. Mughabghab, C.L. Dunford, Phys. Lett. B 487, 155 (2000).

16. B.L. Berman, S.C. Fultz, Rev. Mod. Phys. 47, 713 (1975).

17. V.A. Erokhova, M.A. Elkin, A.V. Izotova, B.S. Ishkhanov, I.M. Kapitonov, E.I. Lileeva, E.V. Shirokov, Izvestiya RAN. Seriya Fiz. 67, 1479 (2003).

18. V.V. Varlamov, M.E. Stepanov, V.V. Chesnokov, Izvestiya RAN. Seriya Fiz. 67, 656 (2003).

19. V.A. Plujko, O.A. Bezshyyko, I.M. Kadenko, L.O. GolinkaBezshyyko, AIP Conference Proceedings. 769, 1108 (2005), Proc. Intern. Conf. Nucl. Data Sci. Techn., Santa Fe, New Mexico, 26 Sept.-1 Oct. 2004, edited by. R.C. Haight, M.B. Chadwick et al., (Melville, New York, 2005).

20. V.A. Plujko, I.M. Kadenko, O.A. Bezshyyko, L.O. GolinkaBezshyyko, O.I. Davidovskaya, Int. J. Mod. Phys. E 15, 387 (2006). 